\title{
Microfour-point probe for studying electronic transport through surface states
}

Petersen, Christian Leth; Grey, Francois; Shiraki, I.; Hasegawa, S.

\section{Published in:}

Applied Physics Letters

Link to article, DOI:

$10.1063 / 1.1329871$

Publication date:

2000

Document Version

Publisher's PDF, also known as Version of record

Link back to DTU Orbit

Citation (APA):

Petersen, C. L., Grey, F., Shiraki, I., \& Hasegawa, S. (2000). Microfour-point probe for studying electronic transport through surface states. Applied Physics Letters, 77(23), 3782-3784. https://doi.org/10.1063/1.1329871

\section{General rights}

Copyright and moral rights for the publications made accessible in the public portal are retained by the authors and/or other copyright owners and it is a condition of accessing publications that users recognise and abide by the legal requirements associated with these rights.

- Users may download and print one copy of any publication from the public portal for the purpose of private study or research.

- You may not further distribute the material or use it for any profit-making activity or commercial gain

- You may freely distribute the URL identifying the publication in the public portal

If you believe that this document breaches copyright please contact us providing details, and we will remove access to the work immediately and investigate your claim 


\title{
Microfour-point probe for studying electronic transport through surface states
}

\author{
C. L. Petersen and F. Grey ${ }^{\mathrm{a})}$ \\ Mikroelektronik Centret, Technical University of Denmark, Bldg. 345 East, DK-2800 Lyngby, Denmark
}

I. Shiraki and S. Hasegawa

Department of Physics, School of Science, University of Tokyo, 7-3-1 Hongo, Bunkyo-ku, Tokyo 113-0033, Japan

(Received 13 June 2000; accepted for publication 12 October 2000)

\begin{abstract}
Microfour-point probes integrated on silicon chips have been fabricated with probe spacings in the range 4-60 $\mu \mathrm{m}$. They provide a simple robust device for electrical transport measurements at surfaces, bridging the gap between conventional macroscopic four-point probes and scanning tunneling microscopy. Measurements on $\mathrm{Si}(111)$ surfaces in ultrahigh vacuum reveal that the $\mathrm{Si}(111)-\sqrt{3} \times \sqrt{3}-\mathrm{Ag}$ structure induced by a monolayer of $\mathrm{Ag}$ atoms has a four-point resistance two orders of magnitude lower than that of the $\mathrm{Si}(111)-7 \times 7$ clean surface. We attribute this remarkable difference to direct transport through surface states, which is not observed on the macroscopic scale, presumably due to scattering at atomic steps. (c) 2000 American Institute of Physics.

[S0003-6951(00)02749-2]
\end{abstract}

Clean facets of many crystalline materials exhibit reconstructions of the outer atomic layers, which result in a new two-dimensional band structure at the surface. While the dispersion of these bands can be measured by spectroscopic techniques, the characterization of charge transport in these surface states remains a challenge. ${ }^{1,2}$

Four-point probes with electrode spacings in the millimeter range are not well suited for the study of surface state conductivity, as transport through these states is interrupted by steps and defects on the surface. It is possible to investigate surface state transport indirectly by scanning tunneling microscopy (STM) by studying the scattering of electrons at surface defects. ${ }^{3-6}$ Direct transport measurements, however, require a multiprobe technique.

The microfour-point probes described in this letter were made using silicon-based microfabrication technology following a fabrication procedure similar to that for atomic force microscope probes. The probes consist of four sharpened silicon oxide cantilevers, coated with $\mathrm{Ti}$, extending from a silicon support chip. ${ }^{7}$ The cantilevers are very flexible, so that contacting a surface is straightforward. Electrode spacings of 8 and $20 \mu \mathrm{m}$ were used in the experiments described here.

The microscopic probes were integrated in a customized ultrahigh vacuum (UHV) scanning electron microscopy (SEM) system. Microslides moved the four-point probe into contact with the sample surface while the approach was monitored in the UHVSEM, which included an Ag evaporation cell to produce the $\mathrm{Si}(111)-\sqrt{3} \times \sqrt{3}-\mathrm{Ag}$ reconstruction. The microfour-point probe was connected to a custom dc measurement system (current source and a high-precision instrumentation amplifier). A current $I$ applied over the outer electrodes produces a voltage drop $V$ over the inner probes, the four-point resistance defined as $R=V / I$.

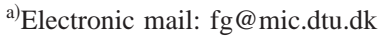

The samples were $20 \times 3 \mathrm{~mm}^{2} n$-type $\mathrm{Si}(111)$ with a nominal resistivity of $10-100 \Omega \mathrm{cm}$. The sample surfaces were patterned to generate large terraces during flashing. The patterning was done using a laser etching facility. ${ }^{8}$ To make large step-free terraces, grids of micron-sized small holes were etched with the laser, similar to the patterns used by Ogino. ${ }^{9}$ Grid spacings of 5, 10, 15, and $20 \mu \mathrm{m}$ were used. The samples were heated resistively at $1250{ }^{\circ} \mathrm{C}$ in intervals of 10-60 s, while the chamber pressure was kept below 5 $\times 10^{-9}$ Torr. The flashing procedure created step bunching due to electromigration. After a total of $3000 \mathrm{~s}$ of flashing, the step bunches were roughly aligned to the position of the original hole grid.

The two surface reconstructions investigated in this study were the $\mathrm{Si}(111)-7 \times 7$ and the $\mathrm{Si}(111)-\sqrt{3} \times \sqrt{3}-\mathrm{Ag}$. Both have a surface state at the Fermi energy, and should thus be conducting. However, the dispersion of the half-filled state on the $\mathrm{Si}(111)-7 \times 7$ surface is very low, ${ }^{10}$ and recent STM measurements indicate that this surface has a low conductance. ${ }^{6}$ The $\mathrm{Si}(111)-\sqrt{3} \times \sqrt{3}-\mathrm{Ag}$ surface, on the other hand, has a band with strong dispersion at the Fermi level. ${ }^{2}$ Macroscopic four-point measurements showed a $10 \%$ difference in conductance between the two reconstructions. ${ }^{11}$

The position of single atomic steps was revealed by deposition of around 0.1 monolayers of $\mathrm{Ag}$, which decorates step edges. It was thus confirmed that atomically flat terraces were created between the step bunches. The microscopic four-point probe was then brought into contact with the sample, as shown in Fig. 1(b). The total probe width is larger than the width of the terrace, so the electrodes are positioned on neighboring terraces.

Measurements on the $\mathrm{Si}(111)-7 \times 7$ and $\mathrm{Si}(111)-\sqrt{ } 3$ $\times \sqrt{3}-\mathrm{Ag}$ surfaces are shown in Fig. 2. These measurements were performed with a probe with $20 \mu \mathrm{m}$ electrode spacing in a region with $15 \mu \mathrm{m}$ spaced step bunches. The four-point probe resistance was $24 \mathrm{k} \Omega$ on the $7 \times 7$ surface and $280 \Omega$ on the $\sqrt{3} \times \sqrt{ } 3$ surface. The measured values were reproduced 

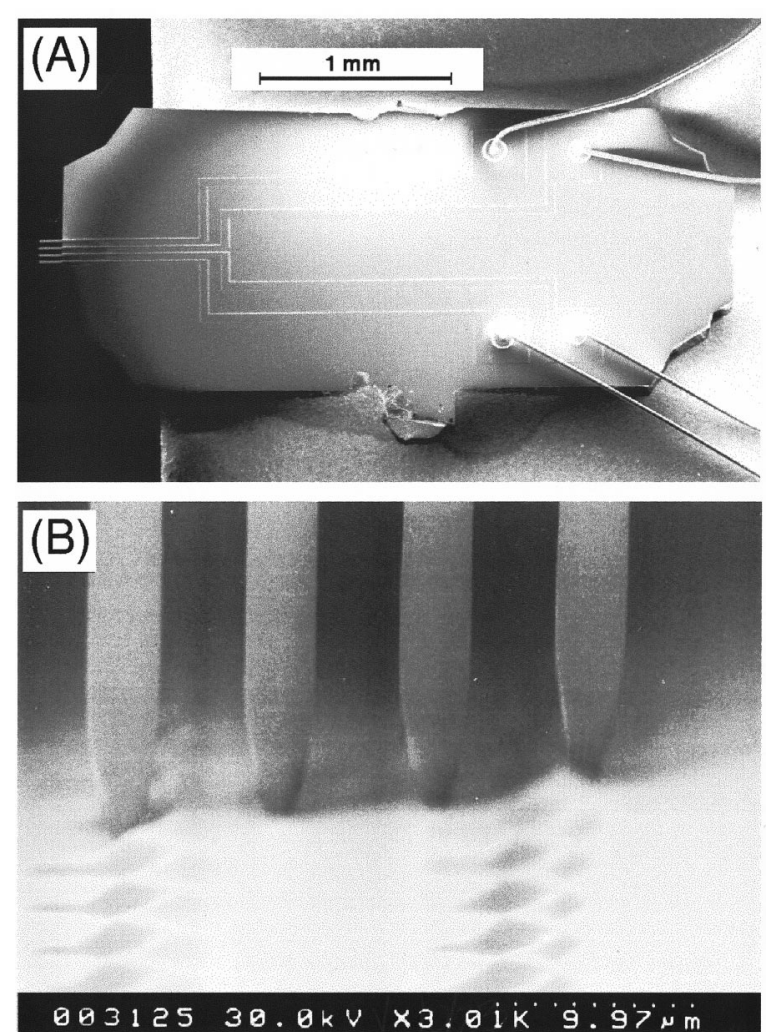

FIG. 1. (a) SEM image of a microscopic four-point probe. The microcantilevers are connected electrically to bonding pads on a silicon substrate. Thin gold wires are bonded from these pads to the external measurement circuitry. (b) UHVSEM image showing a microfour-point probe with an electrode spacing of $8 \mu \mathrm{m}$ positioned with the inner two electrodes on a single terrace on a $\mathrm{Si}(111)-7 \times 7$ surface

with two different probes on two different (111) substrates before and after Ag deposition. The difference between the results for the $7 \times 7$ and $\sqrt{3} \times \sqrt{3}$ reconstructions, nearly two orders of magnitude in four-point resistance, is remarkably large compared with the $10 \%$ difference measured with macroscopic probes. ${ }^{11}$
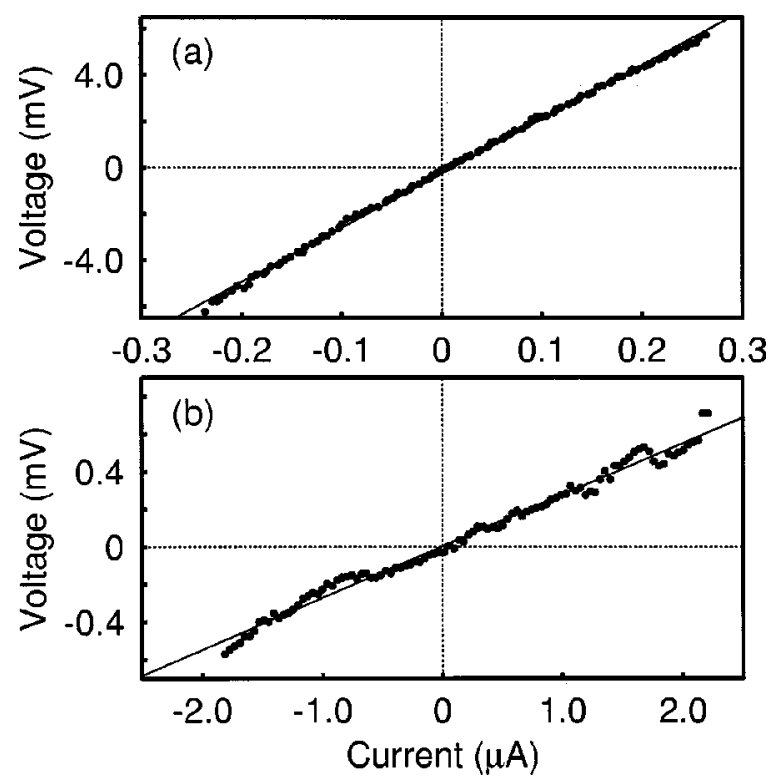

FIG. 2. Linear $I-V$ curves obtained with a microfour-point probe with 20 $\mu \mathrm{m}$ electrode spacing on the $\mathrm{Si}(111)-7 \times 7$ surface (a) and the $\mathrm{Si}(111)-\sqrt{3}$ $\times \sqrt{3}-$ Ag surface $(b)$ on the same sample.

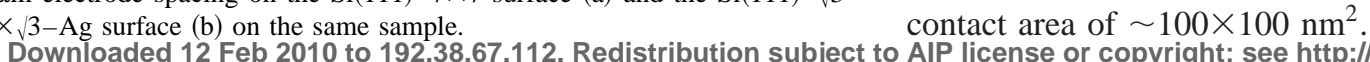

We suggest two possible explanations. One is that a larger fraction of the current runs in the space charge region for the microscopic probe and thus the effect of the space charge layer is more significant in the microscopic case. This is mainly a geometrical effect. The other possible explanation is that a larger fraction of the current runs in the surface states.

Concerning the space charge layer, we note that the clean $\operatorname{Si}(111)$ surface has the Fermi level pinned at $0.65 \mathrm{eV}$ above the valence-band maximum. ${ }^{12}$ For an $n$-type $100 \Omega \mathrm{cm}$ silicon crystal, the bulk Fermi level is approximately $0.75 \mathrm{eV}$ above the valence-band maximum. ${ }^{13}$ The band bending beneath the $7 \times 7$ surface is thus only about $0.1 \mathrm{eV}$, and so the space charge layer region has a conductance close to that of the bulk. Under these conditions, the sample can be approximated as a semi-infinite system with a uniform homogenous conductance. In this limit, the bulk resistivity $\rho$ can be calculated from the four-point probe resistance $R$ as $\rho$ $=2 \pi s R$, where $s$ is the electrode spacing of the probe. ${ }^{14} \mathrm{~A}$ resistivity value of $295 \Omega \mathrm{cm}$ is deduced, which is comparable with the nominal bulk resistivity of the sample, and hence consistent with the semi-infinite approximation.

Since the four-point probe resistance falls two orders of magnitude after deposition of a monolayer of $\mathrm{Ag}$, we can conclude that the surface channel effectively shorts out the bulk channel in this case. The Fermi level for the $\sqrt{3} \times \sqrt{ } 3$ surface is pinned at a position $0.1 \mathrm{eV}$ above the valence-band maximum, ${ }^{2}$ and thus the band bending is in this case 0.65 $\mathrm{eV}$. The electrode spacing is considerably larger than the space charge layer thickness, as estimated from the nominal bulk resistivity. Therefore, we can approximate the space charge layer as an infinite two-dimensional sheet, and the surface sheet resistivity $R_{s}$ can then be extracted for the $\sqrt{ } 3 \times \sqrt{3}$ surface as $R_{s}=R \pi / \ln 2$. This yields a sheet resistance of $1.2 \times 10^{3} \Omega$, or a corresponding conductance of 8.0 $\times 10^{-4} \Omega^{-1}$. For comparison, macroscopic four-point measurements by Hasegawa et al. show a difference in conductance between the $\sqrt{3} \times \sqrt{3}$ and the $7 \times 7$ surfaces of about $1.2 \times 10^{-4} \Omega^{-1}{ }^{2}$ In Fig. 3, the microscopic and macroscopic results are displayed together with a curve depicting the theoretical variation of the space charge layer conductance $\sigma_{\mathrm{sc}}$ as a function of band bending, calculated from the bulk carrier mobilities $\mu_{n}, \mu_{p}$ and the excess carrier densities $\Delta N, \Delta P$ in the space charge layer ${ }^{15}$

$$
\sigma_{\mathrm{sc}}=e \mu_{n} \Delta N+e \mu_{p} \Delta P .
$$

The surface conductance of the $\sqrt{ } 3 \times \sqrt{ } 3$ surface as measured with the microscopic four-point probe is nearly one order of magnitude higher than expected from band bending alone. We therefore conclude that the extra conductance measured with the microfour-point probe on the $\sqrt{3} \times \sqrt{3}$ surface is due to conduction directly through the surface states.

We suggest two possible explanations for obtaining this result on the microscopic scale. One is that the scattering in the surface state channel is reduced due to the smaller number of atomic steps between the voltage probes. The other explanation is improved electrical contact to the surface states using the flexible microcantilever electrodes. Tests show that the electrodes leave only minimal damage over a 


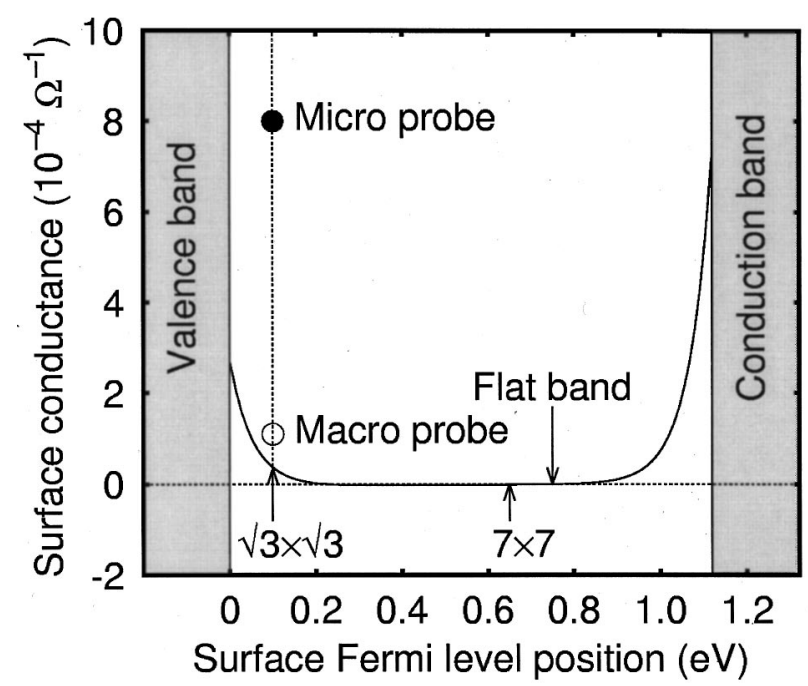

FIG. 3. The solid line shows the calculated space charge layer conductance as a function of the Fermi level position. The closed circle represents the measurement with the microfour-point probe, and the open circle represents a previous measurement performed with a macroscopic four-point probe (Ref. 2).

An estimate of the conductivity of the surface states can be deduced from the difference in conductance between the microprobe measurement and theoretical prediction for band bending alone, $7.6 \times 10^{-4} \Omega^{-1}$, and the typical thickness of a reconstructed surface, $1 \mathrm{~nm}$. This yields a resistivity of $1.3 \times 10^{-4} \Omega \mathrm{cm}$, comparable with the resistivities of bulk metals such as bismuth. The $\sqrt{3} \times \sqrt{3}$ structure has no relationship to metallic silver. ${ }^{16}$ Therefore, we emphasize that this metallic conductivity is not due to a thin metallic film, but is an intrinsic property of a surface reconstruction.

A more detailed theoretical analysis of this result will require modeling four-point probe measurements in realistic systems (not semi-infinite or planar), ${ }^{17}$ and including effects of step structure and finite contact area. In addition, comparison with theory will require a complete mapping of the band structures of these surfaces.
The technique presented here can clearly be extended to study other surface reconstructions, ${ }^{17}$ each having potentially unique electronic transport properties. The microfour-point probe is thus a useful addition to local probe techniques for applications in surface science and technology.

The authors are supported by the International Collaboration Program (No. 11694059) in Grants-in-Aid for Scientific Research from the Ministry of Education, Science, Sports and Culture of Japan. Two authors (I.S. and S.H.) are also supported by the Creative Basic Research Program (No. 09NP1201) and by a CREST Project of The Japan Science and Technology Corporation.

${ }^{1}$ M. Henzler, in Surface Physics of Materials, Vol. 1, edited by J. M. Blakeley (Academic, New York, 1975), pp. 241-278.

${ }^{2}$ S. Hasegawa, X. Tong, C.-S. Jiang, Y. Nakajima, and T. Nagao, Surf. Sci. 386, 322 (1997)

${ }^{3}$ P. Avouris, I.-W. Lyo, and Y. Hasegawa, IBM J. Res. Dev. 39, 603 (1995).

${ }^{4}$ E. J. Heller, M. F. Crommie, C. P. Lutz, and D. M. Eigler, Nature (London) 369, 464 (1994).

${ }^{5}$ N. Sato, S. Takeda, T. Nagao, and S. Hasegawa, Phys. Rev. B 59, 2035 (1998).

${ }^{6}$ S. Heike, S. Watanabe, Y. Wada, and T. Hashizume, Phys. Rev. Lett. 81, 890 (1998).

${ }^{7}$ C. L. Petersen, thesis, Mikroelektronik Centret, Technical University of Denmark, 1999.

${ }^{8}$ M. Mullenborn, H. Dirac, and J. W. Petersen, Appl. Surf. Sci. 86, 568 (1995).

${ }^{9}$ T. Ogino, Surf. Sci. 386, 137 (1997).

${ }^{10}$ P. Martenson, W.-X. Ni, G. V. Hansson, J. M. Nicholls, and B. Reihl, Phys. Rev. B 36, 5974 (1987).

${ }^{11}$ C.-S. Jiang, S. Hasegawa, and S. Ino, Phys. Rev. B 54, 10389 (1996)

${ }^{12}$ J. E. Demuth, W. J. Thompson, N. J. DiNardo, and R. Imbihl, Phys. Rev. Lett. 56, 1408 (1996).

${ }^{13}$ S. M. Sze, Semiconductor Devices, Physics and Technology (Wiley, New York, 1985).

${ }^{14}$ F. M. Smits, Bell Syst. Tech. J. 37, 711 (1958).

${ }^{15}$ C. E. Young, J. Appl. Phys. 32, 329 (1961).

${ }^{16}$ V. G. Lifshits, A. A. Saranin, and A. V. Zotov, Surface Phases on Silicon (Wiley, New York, 1994).

${ }^{17}$ D. W. Koon and C. J. Knickerbocker, Rev. Sci. Instrum. 69, 3625 (1998). 